\title{
Electrostatic stretching of grafted maleic acid copolymer chains
}

\author{
L. Renner ${ }^{1}$ T. Pompe ${ }^{1 *}$, C. Werner ${ }^{1,2,3}$ \\ ${ }^{1}$ Leibniz Institute of Polymer Research Dresden, Max Bergmann Center of Biomaterials Dresden, Hohe Str. 6, \\ 01069 Dresden, Germany \\ ${ }^{2}$ Center for Regenerative Therapies Dresden, Germany \\ ${ }^{3}$ Institute of Biomaterials and Biomedical Engineering, University of Toronto, Toronto, Ontario, Canada
}

Received 3 November 2008; accepted in revised form 9 December 2008

\begin{abstract}
We report on the swelling behaviour of thin maleic acid copolymer films in dependence of $\mathrm{pH}$ and ionic strength. The response of the polymer films was studied by quartz crystal microbalance with dissipation monitoring. It was found that solution's $\mathrm{pH}$ and ionic strength can be separately used to adjust the swelling of the maleic acid copolymer films. While the $\mathrm{pH}$ affects the degree of dissociation of the maleic acid groups, ionic strength triggers the interaction of the resulting charges along the polymer chains. The dominance of electrostatic interactions in the system was obvious from the maximal extension of the grafted polymer chains at very low ionic strength. Tuning the expansion of grafted polymer chains by $\mathrm{pH}$ and ionic strength can be utilized in various applications of biointerfacial research to adjust the physicochemical properties of polymer thin film coatings.
\end{abstract}

Keywords: coatings, swelling, grafting density, ionic strength, $Q C M-D$

\section{Introduction}

Maleic acid copolymer films are versatile polymer platforms [1] that can be applied to study many aspects in biointerfacial science such as blood compatibility [2], protein adsorption [3], cell and tissue engineering $[4,5]$, and supported lipid bilayer membranes [6]. This versatility stems from several facts: the maleic anhydride is highly reactive towards primary amines and alcohols, the maleic acid functionalities can be reversibly converted into maleic anhydride moieties, and the density of anhydride moieties can be adjusted by the choice of the comonomer [1]. The reactivity of the anhydride groups offers exciting options for the covalent attachment of proteins and other biomolecules while the hydrolyzed copolymers allow for the physical adsorption of biomolecules with different anchorage strength $[1,3,5]$.

The kinetic and static swelling of maleic acid copolymer films in phosphate buffered saline (pH 7.4 and $\mathrm{pH} 3$ ) was subject of an earlier study [7]. It was found that the different time stages of swelling could be described according to polymer theory [8] of free polymer chains pointing to a very low number of grafting points per polymer chain. The low number of grafting points was also supported by the observed scaling laws in dependence on molecular weight of the polymer chains at high and low $\mathrm{pH}$. These scaling laws correlated very well to swelling of free polymer chains in good and bad solvent conditions $[9,10]$. An intermediate swelling step was found to be affected by the chemical conversion of the maleic anhydride to the

*Corresponding author, e-mail: pompe-tilo@ipfdd.de

(C) BME-PT and GTE 
maleic acid moieties which accelerated the swelling kinetics. The swelling kinetics of polymer films readily accounted for the variation in hydrophilicity and polarity of the polymer chains due to the variation of the comonomer and were in excellent agreement with swelling studies of maleic anhydride copolymers in solution [11].

The response of the copolymer films to varying properties of aqueous environments is of crucial importance for intended further applications in biointerfacial science [14]. In addition to the variation of the $\mathrm{pH}$ changes of the ionic strength often occur and need to be considered when applying thin polymer films e.g. in biosensor devices to gain the optimum activity of immobilized enzymes within a copolymer-supported lipid bilayer membrane. Therefore we set off to address the question how maleic acid copolymer films swell at varied ionic strength. The swelling behavior was analyzed for buffer solutions across a broad range of different ionic strength at $\mathrm{pH} 7$ using a quartz crystal microbalance with dissipation monitoring. The observed layer thickness changes revealed an electrostatically driven expansion of the grafted polymer chains causing almost fully stretched polymer molecules at the conditions of maximum repulsion.
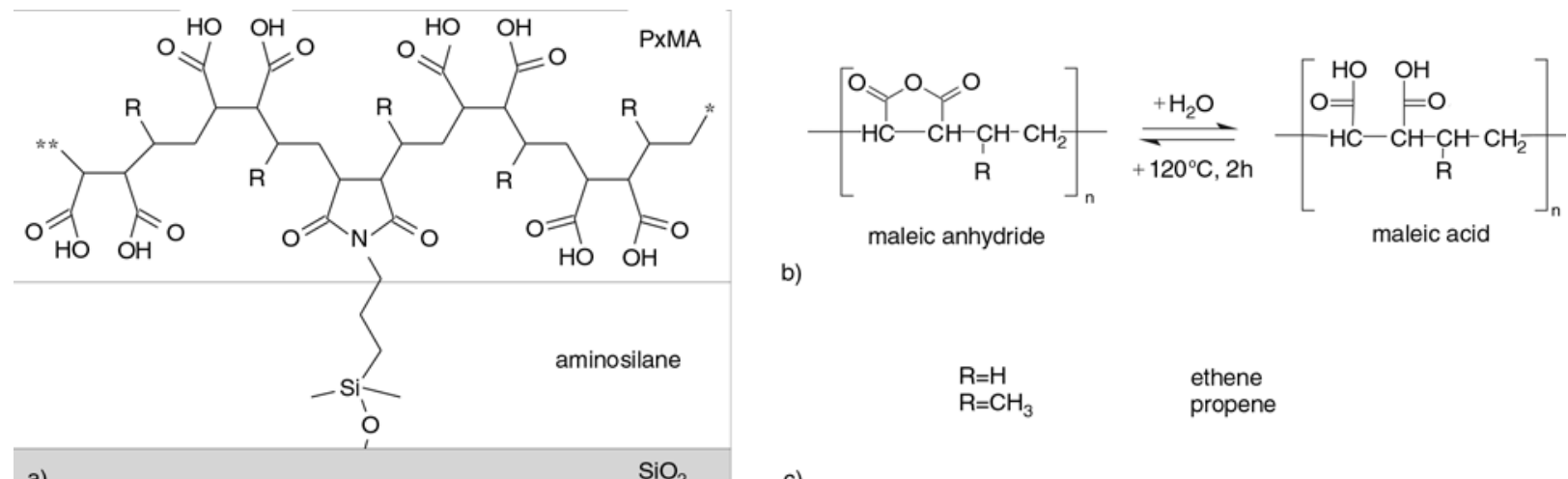

b)

$$
\begin{array}{ll}
\mathrm{R}=\mathrm{H} & \text { ethene } \\
\mathrm{R}=\mathrm{CH}_{3} & \text { propene }
\end{array}
$$

c)

Figure 1. Schematic view of the covalently immobilized maleic acid copolymer layers (a), the reversible hydrolysis between maleic acid and anhydride functionalities (b). The side chains $R$ vary with the type of comonomer unit (propene or ethene) (c).

Table 1. A summary of maleic anhydride copolymer film properties taken from $[1,7]$

\begin{tabular}{|l|c|c|}
\hline & PPMA & PEMA \\
\hline RMS roughness $^{1}$ & $0.34 \mathrm{~nm}$ & $0.8 \mathrm{~nm}$ \\
\hline thickness $^{2} \mathrm{pH} 3( \pm 0.5 \mathrm{~nm})($ hydrolyzed $)$ & $20 \mathrm{~nm}$ & $45 \mathrm{~nm}$ \\
\hline COOH group surface density $^{3}$ & $7 \cdot 10^{14} \mathrm{~cm}^{-2}$ & $1.2 \cdot 10^{15} \mathrm{~cm}^{-2}$ \\
\hline water contact angle $\left.^{4} \pm 3 \mathrm{deg}\right)$ & $38^{\circ}$ & $26^{\circ}$ \\
\hline isoelectric point & \\
\hline
\end{tabular}

${ }^{1}$ determined by scanning force microscopy; ${ }^{2}$ ellipsometry measurements; ${ }^{3}$ determined by XPS after methionine amide conversion; ${ }^{4}$ measurement of hydrolyzed copolymer surfaces; ${ }^{5}$ electrokinetic measurements 
the PEMA solution concentrations were adjusted to $0.03,0.1$, and $0.3 \%$. The polymer films were thoroughly characterized with respect to water contact angle, film thickness, surface roughness, and chemical composition in dry and wet conditions, as recently published $[1,7]$. A schematic of the polymer platform is given in Figure 1. Important film properties are summarized in Table 1.

\subsection{Quartz Crystal Microbalance with Dissipation Monitoring (QCM-D)}

Swelling studies were performed on a QCM-D 300 (Q-Sense $\mathrm{AB}$ ) with dissipation monitoring. The method is described in detail elsewhere [12]. In short, frequency changes are directly correlated to mass changes. Dissipation is attributed to dissipative energy losses of the attached layers on an oscillating crystal surface. The frequency and dissipation data were fitted to the Voigt-Voinova model to calculate the corresponding thickness changes [13].

\subsection{Swelling studies}

To test the influence of different ionic strengths and $\mathrm{pH}$ on the thickness of polymer films distinct solutions have been applied. The substrates have been immersed in HEPES (4-(2-hydroxyethyl)-1-piperazineethanesulfonic acid) buffer in order to change the ionic strength, HEPES buffer ( $\mathrm{pH}$ 7.2) was diluted 1:10 (10-1 HEPES), 1:100 (10-2 HEPES), or 1:1 000 (10-3 HEPES) at room temperature. Swelling of the thin copolymer films was further determined in dependence on the solution's $\mathrm{pH}$ using phosphate buffered saline (PBS) at $\mathrm{pH} 7.4$ (Sigma, Munich, Germany) or unbuffered saline solution at $\mathrm{pH} 4$ using the same ionic strength.

\section{Results and discussion}

QCM-D measurements on PPMA and PEMA polymer films in aqueous solutions were performed to unravel the influence of $\mathrm{pH}$ and ionic strength of buffer solutions on the swelling of the polymers. As found in earlier studies [14] switching from low $\mathrm{pH}$ $(\mathrm{pH} 4)$ to high $\mathrm{pH}(\mathrm{pH} 7)$ increases the charge density along the polymer chain due to an increased dissociation of the carboxylic acid groups. The charged moieties on the polymer chains are shielded by counterions in the solution over distances (Debye screening lengths) that exponentially decrease with increasing ionic strength of the solution. Therefore, we expect a substantially stronger electrostatic repulsion between charged polymer chains at lower ionic strength. It is furthermore hypothesized that the increased electrostatic repulsion between the negatively charged carboxylic acid groups should affect the balance between electrostatic and entropic forces of the polymer chains. Infinitely small solution concentrations of ions $c_{\text {ions }} \rightarrow 0$ may lead to a maximum extension of the polymer chains.

This hypothesis was scrutinized by QCM-D experiments. The effect of ionic strength variations is demonstrated on hydrolyzed PPMA and PEMA films (Figure $2 \mathrm{a}$ and $2 \mathrm{~b}$, respectively). The corresponding thickness values are listed in Table 2 and given in Figure 3. The data show a minor decrease
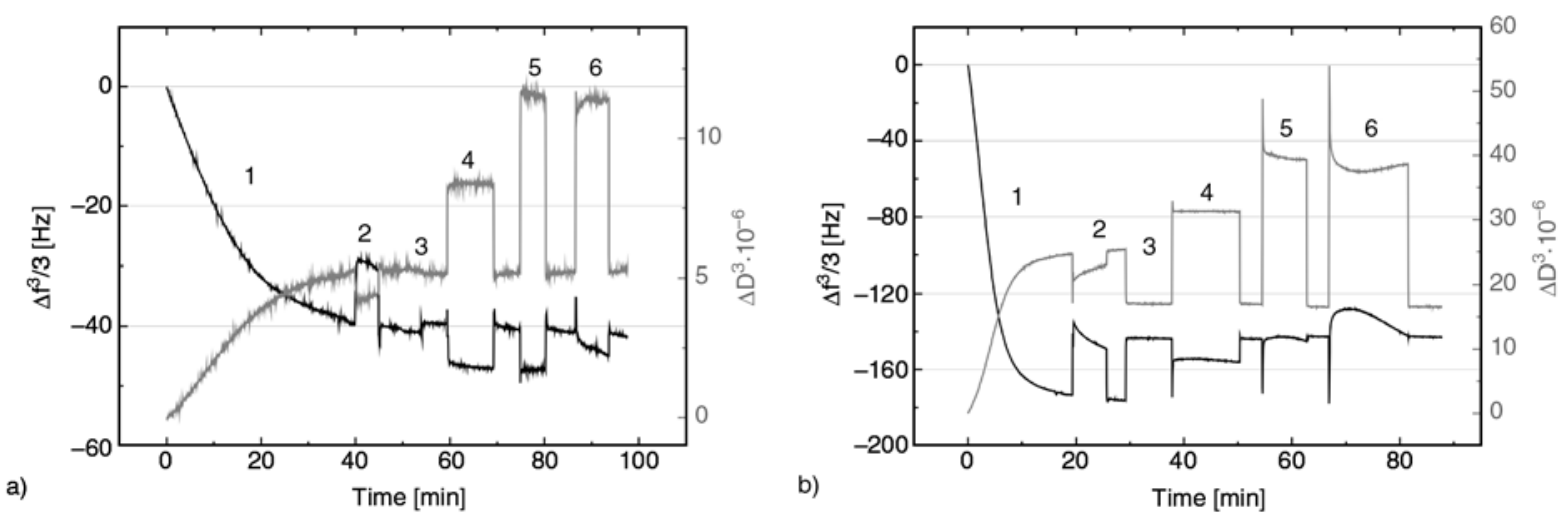

Figure 2. QCM-D measurements on (a) $0.06 \%$ PPMA and (b) $0.1 \%$ PEMA films depending on pH and ionic strength. The conditions have been varied in the following order: (1) swelling in PBS pH 7.4, (2) pH 4 solution, (3) HEPES buffer pH 7.2, (4) 10× diluted HEPES, (5) 100× diluted HEPES and (6) 1 000× diluted HEPES. After each step the solution was recalibrated with HEPES buffer (solution 3). 
Table 2. Thickness variations of copolymer films PPMA and PEMA with various grafting density in dependence of $\mathrm{pH}$ and ionic strength of the solution. For the study of the influence of ionic strength HEPES buffer was diluted 10× (HEPES 10×), 100× (HEPES 10×), and $1000 \times($ HEPES $1000 \times)$

\begin{tabular}{|l|c|c|c|c|c|c|}
\hline & \multicolumn{7}{|c|}{ Thickness [nm] } \\
\hline \multicolumn{1}{|c|}{ Polymer Film } & PBS & pH 4 & HEPES & HEPES 10 $\times$ & HEPES 100x & HEPES 1 000 $\times$ \\
\hline PPMA 0.06\% & 9 & 7 & 9 & 12 & 14 & 14 \\
\hline PEMA 0.03\% & 12 & 8 & 6 & 8 & 14 & 14 \\
\hline PEMA 0.1\% & 34 & 28 & 28 & 36 & 40 & 40 \\
\hline PEMA 0.3\% & 170 & 137 & 150 & 175 & 212 & 225 \\
\hline
\end{tabular}

in the film thickness occurring upon changing from $\mathrm{pH} 7.4$ (PBS) to $\mathrm{pH} 4$ (unbuffered saline solution). This is in excellent agreement with scanning force microscopy data reported earlier [7]. Both $\mathrm{pH}$ solutions closely resembled each other with respect to ion concentration and ionic strength. Hence, the change in the layer thickness can be attributed to the partial protonation of the carboxylic acid groups. As reported in an earlier study [14] the $p K_{a}$ values of the two adjacent carboxylic acid groups of maleic acid $\left(p K_{a 1}=3\right.$ and $\left.p K_{a 2}=7\right)$ explain an only partial dissociation of the acidic groups at $\mathrm{pH}$ 4. Therefore, the electrostatic repulsion along (and between) the grafted polymer chains is lowered at $\mathrm{pH} 4$ which leads to the reported change from good solvent conditions to bad solvent conditions [7].

The impact of electrostatic repulsion on the swelling of the thin polymer films was further shown by changing the ionic strength of the buffer solution. This was accomplished by diluting the HEPES buffer. When changing from PBS to HEPES buffer a slight change in thickness was observed at first, which was attributed to the different electrolyte compositions of the two buffer solutions. The dilution of HEPES buffer led to sudden and significant thickness increases (Table 2). Interestingly, the change from $100 \times$ dilutions to $1000 \times$ dilution did not lead to a further increase in the film thickness, which points to a saturation behaviour: The stretching of the grafted polymer chains was already maximized at the ionic strength of $100 \times$ buffer dilution and the repulsive electrostatic interactions fully overwhelm the entropic contributions. In earlier studies $[1,7]$ PEMA films spin coated from $0.3 \%$ solutions and PPMA films from $0.06 \%$ solutions, respectively, were concluded to contain polymers with very few grafting points per chain only. At these concentrations the grafting-to process occurring during spin-coating resulted in the highest possible packing density with singular grafting points per polymer chain to the substrate. Thus, the layer thickness data of the swollen films can be compared with a theoretical chain length of the polymer molecules. The contour length of an ideal polymer chain with the number of repeating units $N$ (compare Table 1) can be calculated with simple geometrical considerations $D_{\max }=$ $N \cdot l \cdot \cos (\theta / 2)$ assuming no steric hindrance between the polymer chains [15]. For PEMA with a molecular weight of 125000 and PPMA with a molecular weight of 6000 this would lead to a maximum contour length $D_{\max }$ of 506 and $22 \mathrm{~nm}$, respectively, assuming a distance of $1.54 \AA$ and a tetrahedral bond angle of $\theta=68^{\circ}$ between $\mathrm{C}-\mathrm{C}$ bonds [16]. The maximum thickness values of the swollen polymer films correspond to almost one half of the length of a fully stretched polymer chain. As pointed out earlier the polymer chains in the reported case are grafted by very few grafting points to the surface. An estimation for one grafting point per chain which is randomly distributed along the chain would exactly give a mean effective chain length of the grafted chains of one half of the calcu-

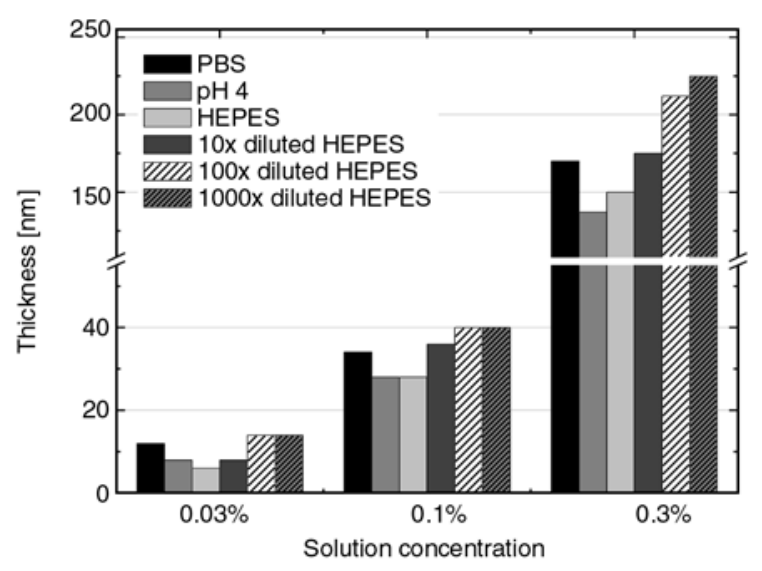

Figure 3. Thickness of the PEMA polymer films as a function of the polymer solution concentration during spin coating (resulting in varied grafting density), the applied $\mathrm{pH}$, and ionic strength 
lated fully stretched polymer chain. Hence, our findings clearly indicate that electrostatic repulsion determines the polymer chain conformation resulting in fully stretched chains at low ionic strength and high $\mathrm{pH}$ values. The results furthermore confirm the very low density of grafting points per polymer chain.

This interpretation is supported by the measurements of polymer films prepared out of different spin-coating concentrations. As found earlier [7] the decreased concentration of polymer chains in the solution leads to a larger available space for the adsorbed molecules on the substrate surface during the spin-coating process. The higher spreading of the adsorbed molecules was found to increase the number of grafting points per polymer chain. Hence, it is expected that the effective free chain length which can expand upon swelling should be lowered. Our results of the PEMA film swelling at low ionic strength perfectly match with this expectation, see Table 2. While the maximum layer thickness was significantly lower than for the highest spin-coating concentration it was again found that in HEPES buffer diluted more than $100 \times$ the film swelling does no longer increase pointing to the maximum possible stretch of the polymer chains.

\section{Conclusions}

In this work different maleic acid copolymer films were analyzed regarding their swelling behaviour in dependence on grafting density and solvent interactions. In aqueous solutions around neutral $\mathrm{pH}$ the maleic acid functionalities are completely de-protonated leading to a high charge density along the polymer chain. Because of that electrostatic repulsion governs the stretching of the grafted polymer chains resulting in a full expansion at low ionic strength. In this context the grafting density of polymer chains on the substrate (affecting the number of grafting sites per chain) modulates the swollen film thickness by variation of the effective free polymer chain length. The reported findings extend our knowledge about important structural characteristics of a versatile set of polymer grafted surfaces.

\section{References}

[1] Pompe T., Zschoche S., Herold N., Salchert K., Gouzy M., Sperling C., Werner C.: Maleic anhydride copolymers - A versatile platform for molecular biosurface engineering. Biomacromolecules, 4, 1072-1079 (2003).

DOI: $10.1021 / \mathrm{bm} 034071 \mathrm{c}$

[2] Sperling C., Salchert K., Streller U., Werner C.: Covalently immobilised thrombomodulin inhibits coagulation and complement activation of artificial surfaces in vitro. Biomaterials, 25, 5101-5113 (2004).

DOI: 10.1016/j.biomaterials.2003.12.014

[3] Renner L., Pompe T., Salchert K., Werner C.: Fibronectin displacement at polymer surfaces. Langmuir, 21, 4571-4577 (2005).

DOI: $10.1021 / \mathrm{la} 046801 \mathrm{n}$

[4] Alberti K., Davey R. E,, Onishi K., George S., Salchert K., Seib F. P., Bornhäuser M., Pompe T., Nagy A., Werner C., Zandstra P. W.: Functional immobilization of signaling proteins enables control of stem cell fate decisions. Nature Methods, 5, 645650 (2008).

DOI: $10.1038 /$ nmeth. 1222

[5] Pompe T., Renner L., Werner C.: Nanoscale features of fibronectin fibrillogenesis depend on protein-substrate interaction and cytoskeleton structure. Biophysical Journal, 88, 527-534 (2005).

DOI: $10.1529 /$ biophysj.104.048074

[6] Renner L., Osaki T., Chiantia S., Schwille P., Pompe T., Werner C.: Supported Lipid bilayers on spacious and $\mathrm{pH}$-responsive polymer cushions with varied hydrophilicity. The Journal of Physical Chemistry B, 112, 6373-6378 (2008). DOI: $10.1021 /$ jp711479f

[7] Pompe T., Renner L., Grimmer M., Herold N., Werner C.: Functional films of maleic anhydride copolymers under physiological conditions. Macromolecular Bioscience, 5, 890-895 (2005). DOI: $10.1002 / \mathrm{mabi} .200500097$

[8] Pitard E., Bouchaud J-P.: Glassy effects in the swelling/collapse dynamics of homogeneous polymers. The European Physical Journal E, Soft Matter, 5, 133-148 (2001) DOI: $\underline{10.1007 / \mathrm{s} 101890170070}$

[9] de Gennes P. G.: Polymers at interfaces: A simplified view. Advances in Colloid and Interface Science, 27, 189-209 (1987).

[10] Binder K.: Scaling concepts for polymer brushes and their test with computer simulation. The European Physical Journal E, Soft Matter, 9, 293-298 (2002). DOI: $10.1140 /$ epje/i2002-10076-2

[11] Rätzsch M., Zschoche S., Steinert V.: Kinetic investigations of reactions with maleic anhydride copolymers and model compounds. Journal of Macromolecular Science, 24, 949-965 (1987). DOI: $\underline{10.1080 / 00222338708076928}$ 
[12] Rodahl M., Höök F., Krozer A., Brzezinski P.: Quartz crystal microbalance setup for frequency and Q-factor measurements in gaseous and liquid environments. Review of Scientific Instruments, 66, 3924-3930 (1995).

DOI: $10.1063 / 1.1145396$

[13] Voinova M. V., Rodahl M., Jonson M., Kasemo B.: Viscoelastic acoustic response of layered polymer films at fluid-solid interfaces: Continuum mechanics approach. Physica Scripta, 59, 391-396 (1999). DOI: 10.1238/Physica.Regular.059a00391
[14] Osaki T., Werner C.: Ionization characteristics and structural transitions of alternating maleic acid copolymer films. Langmuir, 19, 5787-5793 (2003). DOI: $\underline{10.1021 / \mathrm{la} 034439 \mathrm{r}}$

[15] Rubinstein M., Colby R. H.: Polymer physics. Oxford University Press, USA (2003).

[16] Huntley D. R., Markopoulos G., Donovan P. M., Scott L. T., Hoffmann R.: Squeezing $\mathrm{C}-\mathrm{C}$ bonds. Angewandte Chemie International Edition, 44, 7549-7553 (2005).

DOI: 10.1002/anie.200502721 